Jaurnal of Environmental Science, Computer Science and Engineering \& Technology

An International Peer Review E-3 Journal of Sciences and Technology

Available online at www.jecet.org

Section B: Computer Science

Research Article

\title{
Authenticating the Signals for Secured Connections in Virtual Private Network
}

\author{
${ }^{1}$ Douglas, T. Minafa-a and ${ }^{2}$ Kabari, L. Giok \\ Department of Computer Science, Faculty of Natural and Applied Sciences, Post Graduate \\ School, Ignatius Ajuru University of Education, Nigeria.
}

Received: 04 December 2019; Revised: 16 December 2019; Accepted: 18 December 2019

\begin{abstract}
Client-server model for network architecture and connectivity are considered insecure with security threats due to common traffic with exposed rogue devices in Virtual Private Network (VPN). The weaknesses of client-server web application or domain hotspot includes security invasion due to open synchronization among clients or from client device to web or network server. Any unauthorized client can hack client account and can change the data, by pretending to be the legitimate source with masquerading attack or SQL injection. In this paper, architectural design of secure middleware for client-server communication was demonstrated with logical authentication in Virtual Private Network (VPN) to avoid privacy.
\end{abstract}

Keywords: Client, Server, Model, Network, Signal, Authentication, Middleware

\section{INTRODUCTION}

Client-server model is the structural benchmark which is widely adopted for developing distributed web and network applications. Application programming interface (API) provides the functions for creating sockets to facilitate end to end communications over the network. Sockets are the endpoints of logical connections between two hosts and can be used to send and receive data ${ }^{1,2}$. Middleware programming like server-side or object orientation treats socket communications, as much as it treat input and output operations; hence programs can read from or write to sockets, the same way they 
read from or write to the files ${ }^{1}$. To invoke server connection, a server socket should be created and assigned to a port, so that server relate to connections. The port identifies the Transmission Control Protocol service on the socket. For instance, email server uses port 25, while the web server normally runs on port 80.

Consequently, a reliable and secure middleware programming which can perform multithreaded client server communication using Transport Control Protocol (TCP) is quite essential. Security is the key factor communicating privately over a wireless network, hash function with salt is necessary for the database server to ensure scalability and flexibility, as well as high performance, high availability, strong data protection, web and data ${ }^{2}$.

However, the main weakness of client-server network application is that there is no security provided to data which is transferred between clients or from client device to web or network server. Any unauthorized client can hack client account and can change the data, by pretending to be the legitimate source with masquerading attack or SQL injection. Hence, a secure middleware programming which can perform user's authentication and anti-SQL injection on client server signal in Virtual Private Network (VPN) is the aim of this paper.

\section{RELATED WORKS}

In computing perspective, client-server is a system architectural model constituting two entities; the client and server, both communicating over a computer network or on the same computer. Ming and Changjun $^{3}$ opined that web messaging is the recent and very popular incarnation for signal synchronization. The client-server model according to NIST $^{4}$ was originally created to allow many users in sharing access to database applications. Client-server is a typical approach to managing secured network applications. In contrast, peer-to-peer networking, models all devices with equivalent capability rather than specialized controller.

Compared to client-server, a peer to peer network offers some benefits like flexibility in growing the system to accommodate multiple clients. Client-server security model offer advantages in keeping data secure. A system running program that grants requested services from one or more clients is called server machine.

Generally, programs running on client machines make requests to central network controller running as server machine. Networking security services provided by the transport layer is part of Internet application often called TCP/IP (Transport Control Protocol/Internet Protocol) stack ${ }^{2}$.

The transport layer has two types of protocol, Transport Control Protocol and User Datagram Protocol. The most widely used programming interfaces for these protocols are sockets ${ }^{5}$. Sockets provide an interface for security programming at network transport layer.

\section{METHODOLOGY}

The prototyping approach was adopted in developing a network application for authentication of clients' signal in Virtual Private Network (VPN), using Integrated Development Environment for Extensible Hyper Text Mark-up Language (XHTML), Home Page Preprocessor (PHP), MySQL database/ sequential storage with text data and WAMP server for local testing to evaluate the performance; the result showed that privacy invasion can be avoided ${ }^{8,9}$. 


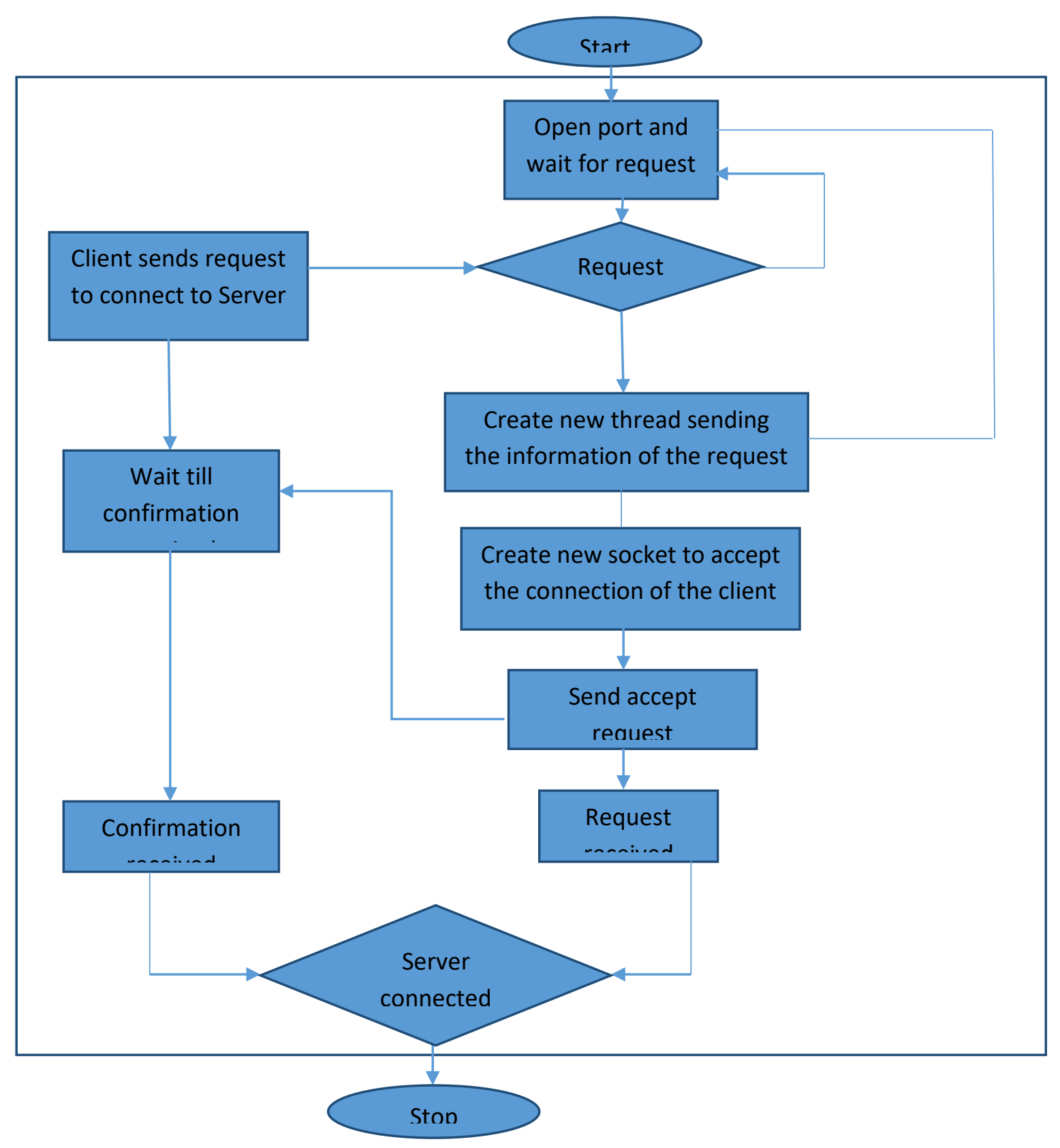

Fig. 1: Signal Authentication for Client-Server in Virtual Private Network (VPN)

\section{RESULTS AND DISCUSSION}

The proposed model has been carefully tested and the result tallied with the expected output; ensuring accessibility due to relatively reduced cost. Valid and invalid data was also considered during evaluation, to ascertain the security strength and validation mechanism; results of various operations are conveyed in the performance report as depicted through the figures below. 


\title{
Registered Client:-Login Here.
}

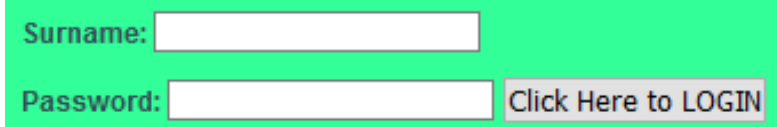

(Fig .2i)

\section{Exd localhost / localhost/ client_se $x$ +}

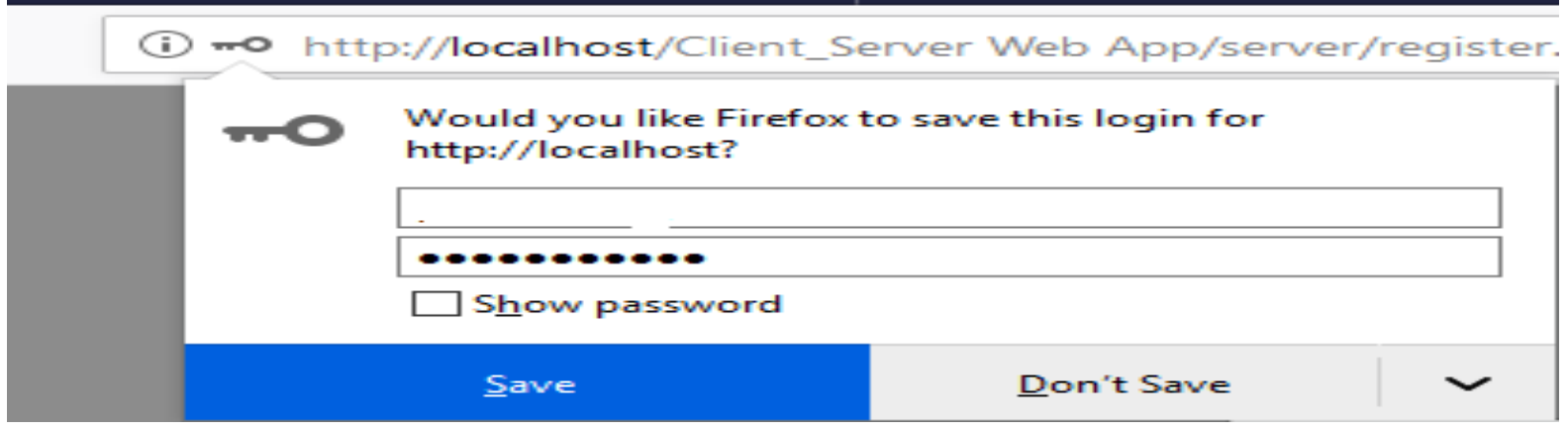

(Fig .2ii)

Your Password Does Not Match Re-Type

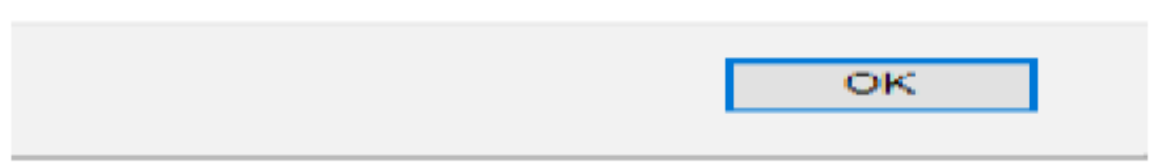

(Fig .2iii)

\section{Din localhost/localhost/dient - $x+$}

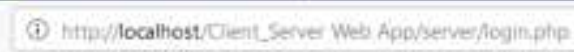

$\cdots \oplus$ 욤

\section{Error Encountered! ....Unauthorized User or Non-Registered Client $>$}

\author{
(Fig .2iv)
}

Fig. 2i-iv: Domain Hotspot and Login Error due to Credential Mismatch 


\section{LOGGEDOUT!}

\section{- Bye, See You Next Time}

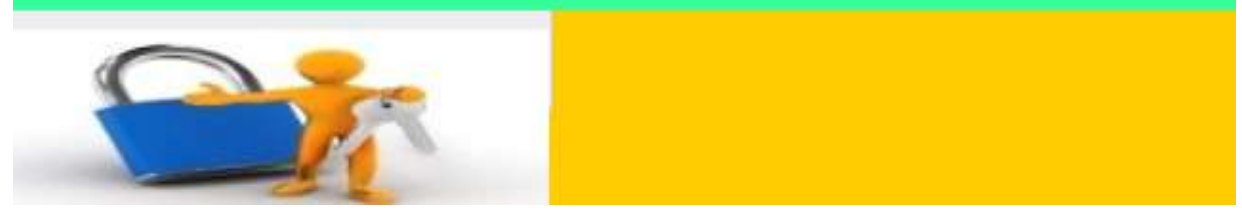

Fig. 3: Log out Interface for Closed Session

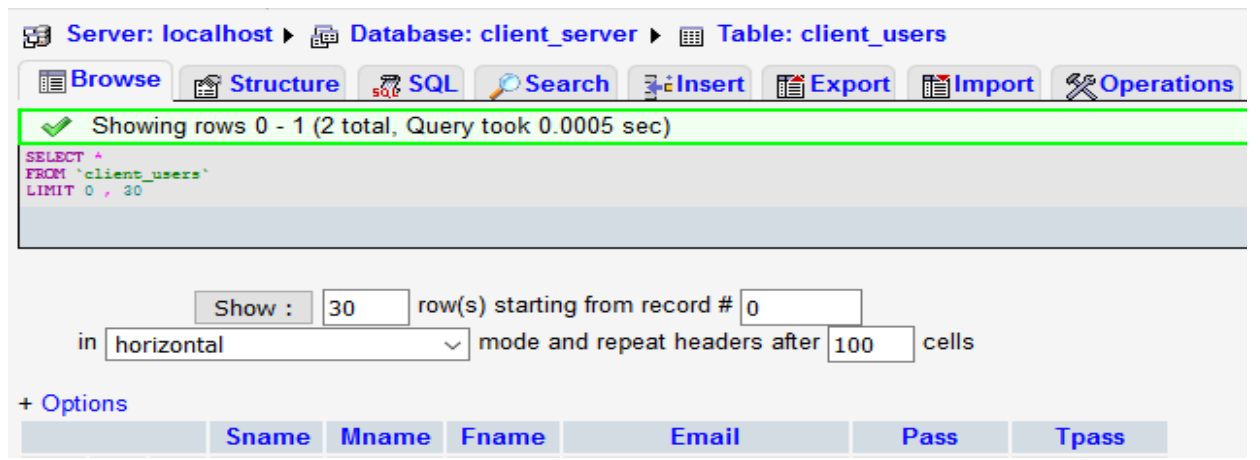

Fig. 4: Clients' database for registering users in MySQL

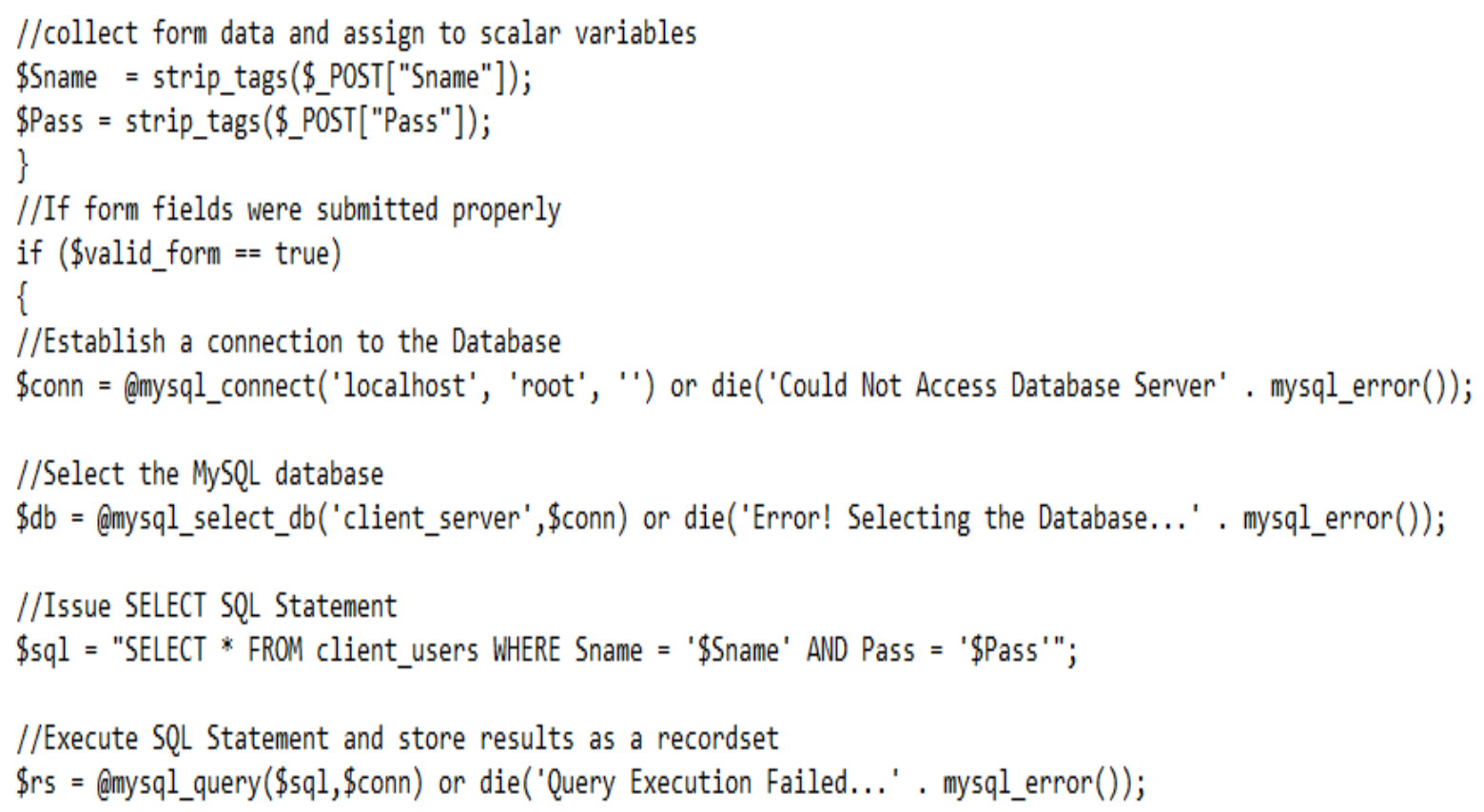

Fig. 5: Middleware script for security against SQL injection 


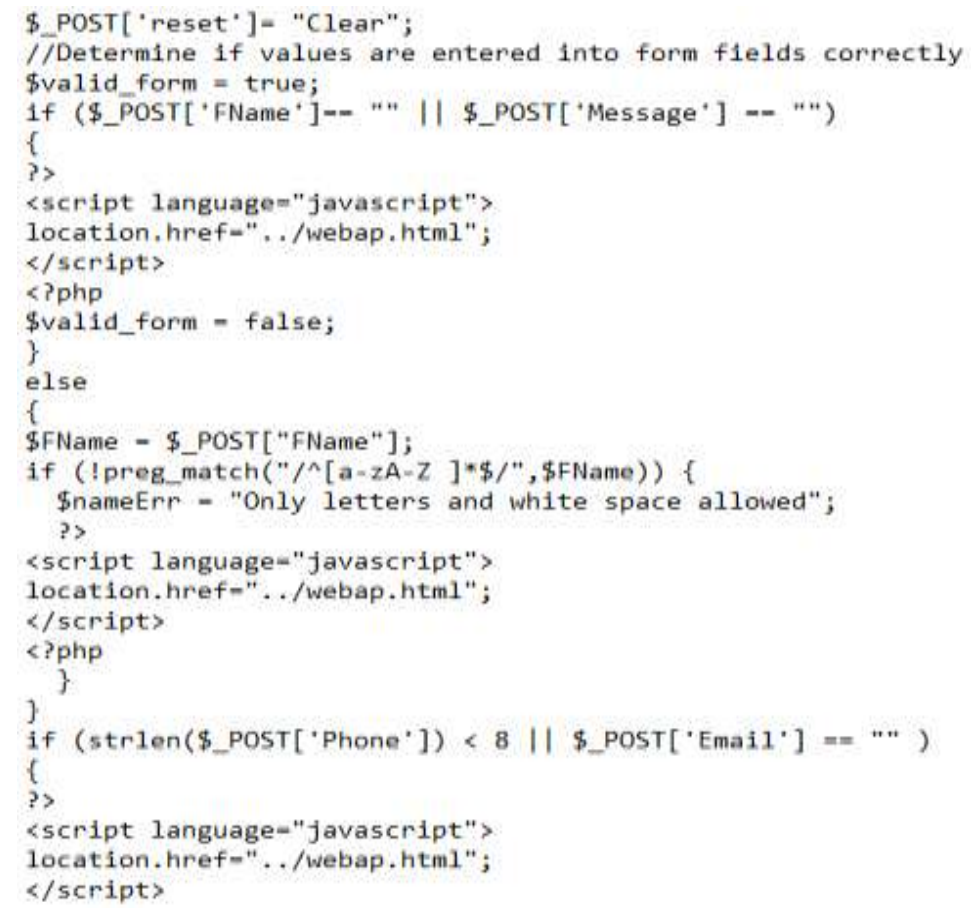

Fig. 6: Middleware script for validating Client's Signal

\section{CONCLUSION}

The prototype was created in a limited time and resources and thus requires optimal implementation for multithread layer in network forum. Many other steps should be taken for enhancement by refining the rule of thumb for adaptation and integration in real time messaging, just as emerging technologies keeps hovering around communication infrastructure coupled with the trends of Internet of Everything (IoE). Then, the network application should be scalable. Before the interaction, the client must establish a connection with the server. In this application, there is one server and client who can communicate to each other via local and testing server.

\section{REFERENCES}

1. M. Arango, A. Dugan, I. Elliott, C. Huitema and S.Pickett, "Media Gateway Control". San Franscisco: Dutch Incorporation, 2008.

2. S.T.Andrew,. "Essentials of Computer Networks and Data Communication". Byblos: Bucharest International Co. 2004.

3. P.C.Mehta \& S. Udani, "Overview of Voice over IP”. Retrieved from URL: <http:// www. cis.upenn.edu / udani/papers/OverviewVoIP.pdf,2006.

4. S.M. Amarande, "Voice over IP security - A Layered Approach". Las Vegas: Brangluise Printing Co.2007.

5. X. Ming, and Z. Changjun, The Socket Programming and Software Design for Communication Based on Client/Server," PACCS '09. 75-77.from URL: <http://www.altera.com /literature /hb/nios2/n2sw_nii5v2.pdf> 2009. 
6. NIST, "Voice over Internet Protocol (VOIP), Security Technical Implementation Guide". Retrieved from URL: <http://csrc.nist.gov/pcig/STIGs/VoIP-STIG-V1R1R-4PDF.pdf> ,2004

7. D.V.Bhatt, S. Schulze, G.P. Hancke,. Secure Internet access to gateway using secure socket layer." ACM Serial Journals, 2006, 55(3), 793-800.

8. B.Goode, "Voice over Internet Protocol (VoIP)". Proceedings of the Institute of Electrical Electronics Engineering, 2004, 9(6), 12-25.

9. R.D.Kuhn, T.J. Walsh and S. Fries, "Security Considerations for Voice over IP Systems". Recommendations of NIST,2004

\section{* Corresponding Author: Douglas, T. Minafa-a;}

Department of Computer Science, Faculty of Natural and Applied Sciences, Post Graduate

School, Ignatius Ajuru University of Education, Nigeria. 